\title{
Dissociation Energy of Molecular Hydrogen Isotopologues
}

\author{
Mariusz Puchalski, Jacek Komasa, and Anna Spyszkiewicz \\ Faculty of Chemistry, Adam Mickiewicz University, Uniwersytetu Poznańskiego 8, 61-614 Poznań, Poland
}

Krzysztof Pachucki

Faculty of Physics, University of Warsaw, Pasteura 5, 02-093 Warsaw, Poland

(Dated: September 6, 2019)

\begin{abstract}
The nonrelativistic energy together with relativistic and quantum electrodynamic corrections for all the molecular hydrogen isotopologues $\left(\mathrm{D}_{2}, \mathrm{~T}_{2}, \mathrm{HD}, \mathrm{HT}, \mathrm{DT}\right)$ were evaluated without expansion in the electron-nucleus mass ratio. The obtained results significantly improve the uncertainty of theoretical predictions, reaching a value below $1 \mathrm{MHz}$ for the total dissociation energy. We observe good agreement with the experimental value for $\mathrm{D}_{2}$ and $3 \sigma$ discrepancy for the HD molecule, while no experimental values for the dissociation energy of molecules involving tritium have yet been obtained.
\end{abstract}

\section{INTRODUCTION}

The dissociation energy of ortho- $\mathrm{H}_{2}$ has recently been measured with sub-MHz uncertainty [1, 2], which is smaller than the contribution due the finite size of the proton. Therefore, for the first time the molecular hydrogen spectroscopy has become sensitive to the nuclear charge radius. Similar progress is expected for HD and $\mathrm{D}_{2}$ systems, which have been measured so far with $10 \mathrm{MHz}$ uncertainty [3, 4]. Moreover, very accurate measurements of several molecular transitions for tritium-containing isotopologues have been obtained [5, 6] very recently, which indicates the possibility of measurements of their dissociation energies in the future.

The current theoretical dissociation energy of para- $\mathrm{H}_{2}$ is $36118.069632(26) \mathrm{cm}^{-1}$ [7], which corresponds to $0.8 \mathrm{MHz}$ of absolute uncertainty. It is in a good agreement with the most recent experimental value of $36118.06945(31) \mathrm{cm}^{-1}$ [8], which will soon be improved [9]. In this work we demonstrate that similar accuracy can be reached for all the other molecular hydrogen isotopologues: $\mathrm{D}_{2}, \mathrm{~T}_{2}, \mathrm{HD}, \mathrm{HT}$, and DT.

The high accuracy of theoretical predictions for molecular levels can only be achieved with the approach, based on nonrelativistic quantum electrodynamic (NRQED) theory. According to NRQED, the total energy of an atom or a molecule can be represented by the expansion in powers of the fine structure constant $\alpha$

$$
E(\alpha)=\sum_{n=2} m \alpha^{n} E^{(n)} .
$$

In our previous work on $\mathrm{H}_{2}$ [7], the first three terms- the nonrelativistic energy $E^{(2)}$, the relativistic correction $E^{(4)}$, and the leading QED correction $E^{(5)}$-were calculated to a high numerical precision in direct four-body variational calculations. The higher order QED corrections were evaluated in the framework of the Born-Oppenheimer (BO) approximation, wherein the $E^{(6)}$ correction was evaluated in a complete way, but $E^{(7)}$ was merely estimated from the dominating terms, which are known from the hydrogen atom [10]. Here, the same method is applied to the heavier homonuclear isotopologues $\mathrm{D}_{2}$ and $\mathrm{T}_{2}$, as well as extended further to heteronuclear systems HD, HT, and DT. The uncertainties assigned to theoretical predictions are below $1 \mathrm{MHz}$, which is an improvement by 1-2 orders of magnitude compared to the most accurate previous results.

\section{NONRELATIVISTIC WAVE FUNCTION}

The quality of the determination of the nonrelativistic wave function is of critical importance for achieving the high accuracy of theoretical results. In the direct nonadiabatic approach, in which all particles are treated on an equal footing, the wave function $\Psi$ is a solution to the four-body Schrödinger equation $H \Psi=E \Psi$ with $E=E^{(2)}$ and the Hamiltonian

$$
\begin{aligned}
& H=T+V \\
& T=\frac{\vec{p}_{0}^{2}}{2 m_{0}}+\frac{\vec{p}_{1}^{2}}{2 m_{1}}+\frac{\vec{p}_{2}^{2}}{2 m}+\frac{\vec{p}_{3}^{2}}{2 m}, \\
& V=\frac{1}{r_{01}}-\frac{1}{r_{02}}-\frac{1}{r_{03}}-\frac{1}{r_{12}}-\frac{1}{r_{13}}+\frac{1}{r_{23}} .
\end{aligned}
$$

The indices 0, 1 denote nuclei, and 2, 3-electrons. In the center-of-mass frame, the wave function $\Psi$ depends only on the interparticle distances $r_{i j}$ and is represented as

$$
\begin{aligned}
\Psi & =\sum_{k}^{N} c_{k} \psi_{k}\left(\vec{r}_{0}, \vec{r}_{1}, \vec{r}_{2}, \vec{r}_{3}\right), \\
\psi_{k} & =\left(1+P_{0 \leftrightarrow 1}\right)\left(1+P_{2 \leftrightarrow 3}\right) \phi_{\{k\}}\left(\vec{r}_{0}, \vec{r}_{1}, \vec{r}_{2}, \vec{r}_{3}\right),
\end{aligned}
$$

where the $P_{i \leftrightarrow j}$ operator accounts for the symmetry with respect to the exchange of nuclei (applicable to homonuclear molecules) or electrons. Two types of explicitly correlated basis functions $\phi_{\{k\}}$ are employed to expand the wave function - the nonadiabatic James-Coolidge (naJC) [11] or the explicitly correlated Gaussian (naECG) [12, 13] basis.

The spatial function within the naJC approach is

$$
\phi_{\{k\}}=e^{-\alpha R-\beta\left(\zeta_{2}+\zeta_{3}\right)} R^{k_{0}} r_{23}^{k_{1}} \eta_{2}^{k_{2}} \eta_{3}^{k_{3}} \zeta_{2}^{k_{4}} \zeta_{3}^{k_{5}}
$$

where $\zeta_{2}=r_{02}+r_{12}, \eta_{2}=r_{02}-r_{12}, \zeta_{3}=r_{03}+r_{13}$, $\eta_{3}=r_{03}-r_{13}$, and $R=r_{01}$. The $\alpha$ and $\beta$ in Eq. (7) denote nonlinear variational parameters, common for the whole 
set of basis functions called 'sector', and $k_{i}$ are non-negative integers collectively denoted as $\{k\}$. If needed, two or more sectors (with different pairs of $\alpha^{(i)}$ and $\beta^{(i)}$ ) can be used. In this work, the naJC basis was employed in calculations of the nonrelativistic energy, which converged up to 13 significant figures. This basis has not been used so far for relativistic calculations, as we have not yet worked out all the integrals needed for matrix elements with relativistic operators.

The Gaussian (naECG) basis, used here in the calculations of the the relativistic and QED corrections, is represented by spatial functions of the form

$$
\phi_{\{k\}}=r_{01}^{n} e^{-a_{k 1} r_{01}^{2}-a_{k 2} r_{02}^{2}-a_{k 3} r_{03}^{2}-a_{k 4} r_{12}^{2}-a_{k 5} r_{13}^{2}-a_{k 6} r_{23}^{2}} .
$$

In the particular case of expectation values of certain relativistic operators, $\phi_{\{k\}}$ is modified to

$$
\begin{aligned}
\phi_{\{k\}}= & r_{01}^{n}\left(1+\frac{r_{23}}{2}\right) \\
& e^{-a_{k 1} r_{01}^{2}-a_{k 2} r_{02}^{2}-a_{k 3} r_{03}^{2}-a_{k 4} r_{12}^{2}-a_{k 5} r_{13}^{2}-a_{k 6} r_{23}^{2}},
\end{aligned}
$$

which ensures that the nonrelativistic wave function exactly satisfies the electron-electron cusp condition [14]. Namely, due to the electron-electron Coulomb interaction, the exact wave function $\Psi\left(r_{23}\right)$ must behave for small $r_{23}$ as $\Psi\left(r_{23}\right) \approx$ $\Psi(0)\left(1+r_{23} / 2\right)$ which is authomatically satisfied in the above basis. The internuclear $r_{01}^{n}$ prefactor enables proper representation of the vibrational part of the wave function. The powers $n$ of this coordinate are restricted to even integers within the range $0-80$ and are generated following the log-normal distribution. The nonlinear $a_{k l}$ parameters are determined variationally in an extensive optimization process. The naECG wave function $\Psi$ has been optimized for a sequence of growing basis set sizes to observe the convergence of the nonrelativistic energy. This convergence is presented in Table \and compared with the results of naJC calculations used here as a benchmark, because they are by far the most accurate ones in the literature. As can be inferred from this table, the naECG nonrelativistic energy is converged to at least 10 significant figures.

\section{THE RELATIVISTIC CORRECTION}

The relativistic correction can be expressed in terms of the expectation value

$$
E^{(4)}=\left\langle\Psi\left|H_{\text {rel }}\right| \Psi\right\rangle
$$

of the Breit-Pauli Hamiltonian $(m=1)$

$$
\begin{aligned}
H_{\mathrm{rel}}= & -\frac{1}{8}\left(p_{2}^{4}+p_{3}^{4}\right)+\frac{\pi}{2} \sum_{x, a}\left(1+\frac{\delta_{s}^{x}}{m_{x}^{2}}\right) \delta^{3}\left(r_{x a}\right) \\
& +\pi \delta^{3}\left(r_{23}\right)-\frac{1}{2} p_{2}^{i}\left(\frac{\delta^{i j}}{r_{23}}+\frac{r_{23}^{i} r_{23}^{j}}{r_{23}^{3}}\right) p_{3}^{j} \\
& +\frac{1}{2} \sum_{x, a} \frac{1}{m_{x}} p_{x}^{i}\left(\frac{\delta^{i j}}{r_{x a}}+\frac{r_{x a}^{i} r_{x a}^{j}}{r_{x a}^{3}}\right) p_{a}^{j} \\
& -\frac{1}{2} \frac{1}{m_{0} m_{1}} p_{0}^{i}\left(\frac{\delta^{i j}}{r_{01}}+\frac{r_{01}^{i} r_{01}^{j}}{r_{01}^{3}}\right) p_{1}^{j}
\end{aligned}
$$

where index $x$ goes over nuclei and $a$ over electrons. The coefficient $\delta_{s}^{x}=0$ for the nuclear spin $s=0$ or 1 , and $\delta_{s}^{x}=1$ for $s=1 / 2$ [15]. In the above formulas, we have omitted all the electron spin-dependent terms because they vanish for the ground electronic state of ${ }^{1} \Sigma_{g}^{+}$symmetry. Moreover, we have omitted also the $p_{x}^{4} /\left(8 m_{x}^{3}\right)$ and $\delta^{3}\left(r_{01}\right)$ terms because their numerical values are smaller than the uncertainty of the whole relativistic correction.

The result for relativistic correction to the dissociation energy $D_{0}$ is shown in Table II $D_{0}$ differs from the expectation values of $H_{\text {rel }}$ by subtraction of the corresponding energy of separated atoms,

$$
E_{x}^{(4)}=-\frac{1}{8}+\frac{1}{4}\left(\frac{1}{m_{x}}\right)^{2}+O\left(\frac{1}{m_{x}}\right)^{3},
$$

and the overall sign. It is worth noting that no term proportional to $1 / m_{x}$ is present in the above formula, so the relativistic recoil correction for separated atoms is of higher order in the mass ratio. Thanks to the regularization of the relativistic operators, which we performed in Ref. [7], and the application of the variational wave function (9), the total relativistic contribution has a very good convergence with the size of the basis set, and the extrapolated values are accurate to at least six digits (see Tab. II).

\section{THE LEADING QED CORRECTION}

The formula for the leading quantum electrodynamic correction $E^{(5)}$ for $\mathrm{H}_{2}$ was obtained in Ref. [7]. However, the nonlogarithmic $\left(m / m_{x}\right)^{2}$ terms are unknown in the case of nuclei with spin $s \neq 1 / 2$. Because their numerical contribution is negligibly small, such terms are absent in the formula employed here 
TABLE I. Convergence of the nonrelativistic energy $E^{(2)}$ (in a.u.) with the increasing size $N$ of the naECG basis set in comparison with the benchmark values from nonadiabatic James-Coolidge (naJC) wave function. The following CODATA 2018 [28] mass ratios were used in these calculations: $m_{\mathrm{p}} / m=1836.15267343(11), m_{\mathrm{d}} / m=3670.48296788(13), m_{\mathrm{t}} / m=5496.92153573(27)$.

\begin{tabular}{cccccc}
\hline \hline$N$ & \multicolumn{1}{c}{$\mathrm{D}_{2}$} & $\mathrm{~T}_{2}$ & $\mathrm{HD}$ & HT & DT \\
\hline 128 & -1.167167911358 & -1.168534104823 & -1.165470991485 & -1.166000790842 & -1.167817701507 \\
256 & -1.167168756439 & -1.168535448080 & -1.165471628967 & -1.166001763875 & -1.167819489839 \\
512 & -1.167168805491 & -1.168535668007 & -1.165471916621 & -1.166002029805 & -1.167819626122 \\
1024 & -1.167168808953 & -1.168535674847 & -1.165471923256 & -1.166002036615 & -1.167819671730 \\
2048 & -1.167168809201 & -1.168535675524 & -1.165471923906 & -1.166002037196 & -1.167819673214 \\
naJC & $-1.16716880928410(5)$ & $-1.16853567573290(8)$ & $-1.16547192396366(5)$ & $-1.16600203732867(6)$ & $-1.16781967343673(5)$ \\
\hline
\end{tabular}

TABLE II. Convergence of relativistic correction to the dissociation energy $D_{0}$ (in $\mathrm{cm}^{-1}$ ) with the increasing size $N$ of the naECG basis set.

\begin{tabular}{cccccc}
\hline \hline$N$ & $\mathrm{D}_{2}$ & $\mathrm{~T}_{2}$ & HD & HT & DT \\
\hline 128 & -0.528337669 & -0.527017169 & -0.52997901 & -0.529443386 & -0.527841985 \\
256 & -0.528218423 & -0.526738994 & -0.52991095 & -0.529374084 & -0.527577232 \\
512 & -0.528201146 & -0.526756712 & -0.52988350 & -0.529372726 & -0.527532246 \\
1024 & -0.528205416 & -0.526750343 & -0.52988661 & -0.529378527 & -0.527524975 \\
2048 & -0.528205935 & -0.526750223 & -0.52988730 & -0.529378110 & -0.527523876 \\
$\infty$ & $-0.52820605(9)$ & $-0.5267500(2)$ & $-0.5298875(2)$ & $-0.5293779(2)$ & $-0.5275236(3)$ \\
\hline \hline
\end{tabular}

$$
\begin{aligned}
E^{(5)}= & -\frac{2 \mathcal{D}}{3 \pi} \ln k_{0}-\frac{7}{6 \pi}\left\langle\frac{1}{r_{23}^{3}}+\sum_{a, x} \frac{m}{m_{x}} \frac{1}{r_{a x}^{3}}\right\rangle_{\epsilon}+\frac{4}{3} \sum_{a, x}\left\{\left(1+\frac{m}{4 m_{x}}+\frac{m^{2}}{m_{x}^{2}}\right) \ln \left(\alpha^{-2}\right)+\frac{19}{30}+\frac{m}{m_{x}} \frac{31}{6}\right. \\
& \left.+\frac{m^{2}}{m_{x}^{2}} \ln \left(\frac{m_{x}}{m}\right)\right\}\left\langle\delta^{3}\left(r_{a x}\right)\right\rangle+\left(\frac{164}{15}+\frac{14}{3} \ln \alpha\right)\left\langle\delta^{3}\left(r_{23}\right)\right\rangle-E_{0}^{(5)}-E_{1}^{(5)}, \\
E_{x}^{(5)}= & -\frac{4}{3 \pi} \frac{\mu_{x}}{m}\left(\ln k_{0}(\mathrm{H})+\ln \frac{\mu_{x}}{m}\right) \\
& +\frac{4}{3 \pi}\left(\frac{\mu_{x}}{m}\right)^{3}\left\{\left(1+\frac{m}{4 m_{x}}+\frac{m^{2}}{m_{x}^{2}}\right) \ln \left(\alpha^{-2}\right)+\frac{19}{30}+\frac{m}{m_{x}}\left(\frac{31}{6}+\frac{7}{2} \ln 2\right)+\frac{m^{2}}{m_{x}^{2}} \ln \frac{m_{x}}{m}\right\},
\end{aligned}
$$

where $\mu_{x}=m_{x} m /\left(m_{x}+m\right)$. The Bethe logarithm is given by [16]

$$
\ln k_{0}=\frac{1}{\mathcal{D}}\langle\vec{J}(H-E) \ln [2(H-E)] \vec{J}\rangle
$$

where

$$
\begin{aligned}
\vec{J} & =\frac{\vec{p}_{0}}{m_{0}}+\frac{\vec{p}_{1}}{m_{1}}-\frac{\vec{p}_{2}}{m}-\frac{\vec{p}_{3}}{m}, \\
\mathcal{D} & =\langle\vec{J}(H-E) \vec{J}\rangle=\mathcal{D}_{0}+\mathcal{D}_{1}, \\
\mathcal{D}_{x} & =\frac{2 \pi}{\mu_{x}^{2}} \sum_{a}\left\langle\delta^{3}\left(r_{a x}\right)\right\rangle,
\end{aligned}
$$

and the following numerical value of the atomic Bethe logarithm is used in the above

$$
\ln k_{0}(\mathrm{H})=2.984128555765498 \text {. }
$$

In the formulas (13)- 18 the expectation values are evaluated with the nonrelativistic wave function $\Psi$, and the notation in Eq. 13 $\langle\ldots\rangle_{\epsilon}$ means the following limit

$$
\left\langle\frac{1}{r_{i j}^{3}}\right\rangle_{\epsilon}=\lim _{\epsilon \rightarrow 0}\left[\left\langle\frac{\theta\left(r_{i j}-\epsilon\right)}{r_{i j}^{3}}\right\rangle+4 \pi(\gamma+\ln \epsilon)\left\langle\delta^{3}\left(r_{i j}\right)\right\rangle\right],
$$

where the symbol $\gamma$ denotes the Euler-Mascheroni constant, and $\theta$ is the Heaviside function.

One subtle point to be clarified is the nuclear self-energy correction and the corresponding definition of the nuclear charge radius. This correction is insignificant for a regular hydrogen atom but non-negligible for muonic hydrogen $(\mu \mathrm{H})$. So, for consistency with the determination of the proton charge radius $r_{p}$ in $\mu \mathrm{H}$ [17], following Ref. [7], we account for this effect in the total energy of the hydrogen molecule in a minimal way, by including in Eq. (13) only logarith- 
mic terms, and the nonlogarithmic terms are absorbed into the mean square nuclear charge radius.

\section{BETHE LOGARITHM}

Since the calculation of the Bethe logarithm $\ln k_{0}$ is the most complicated one, we describe below its evaluation in more detail, extending our previous work [7] to two nuclei with different masses. We express $\ln k_{0}$ in terms of the onedimensional integral [18]

$$
\ln k_{0}=\frac{1}{\mathcal{D}} \int_{0}^{1} d t \frac{f(t)-f_{0}-f_{2} t^{2}}{t^{3}}
$$

with the function $f(t)$ defined as

$$
f(t)=\left\langle\vec{J} \frac{k}{k+H-E} \vec{J}\right\rangle, \quad t=\frac{1}{\sqrt{1+2 k}}
$$

which has the following Taylor expansion

$$
f(t)=f_{0}+f_{2} t^{2}+f_{3} t^{3}+\left(f_{4 l} \ln t+f_{4}\right) t^{4}+O\left(t^{5}\right)
$$

with the coefficients $(m=1)$

$$
\begin{aligned}
f_{0}= & \left\langle J^{2}\right\rangle \\
f_{2}= & -2 \mathcal{D} \\
f_{3}= & \sum_{x} 8 \sqrt{\mu_{x}} \mathcal{D}_{x}, \\
f_{4 l}= & \sum_{x} 16 \mu_{x} \mathcal{D}_{x}, \\
f_{4}= & 4\left\langle\left[\sum_{a, x} \frac{1}{\mu_{x}} \frac{\vec{r}_{a x}}{r_{a x}^{3}}+\left(\frac{1}{m_{0}}-\frac{1}{m_{1}}\right) \frac{\vec{r}_{01}}{r_{01}^{3}}\right]^{2}\right\rangle_{\epsilon} \\
& -2 \sum_{x} \mathcal{D}_{x}\left(1+4 \mu_{x} \ln \frac{\mu_{x}}{4}-4 \mu_{x}\right),
\end{aligned}
$$

which has been obtained from the known high- $k$ expansion by Korobov [20], with all the terms proportional to $\delta^{3}\left(r_{01}\right)$ being neglected. The integrand in Eq. (21), as a smooth function of $t$, was evaluated at 200 equally spaced points in the range $t \in[0,1]$, which enabled relative uncertainty higher than $10^{-7}$. In the numerical calculation of $f(t)$, the resolvent in Eq. (22) was represented in terms of pseudostates of the form $\vec{\phi}^{\Pi}=\vec{r}_{a b} \phi$ for all interparticle coordinates. The nonlinear parameters of $\vec{\phi}^{\Pi}$ were found by a maximization of $f$.

The $f(1)$ value can be determined analytically using the generalized Thomas-Reiche-Kuhn sum rule [19]

$$
\left\langle\vec{J}(H-E)^{-1} \vec{J}\right\rangle=\frac{3}{2}\left(\frac{m}{\mu_{0}}+\frac{m}{\mu_{1}}\right),
$$

which enables an assessment of the completeness of the pseudostates space and the uncertainty estimation. For the given size $N$ of the wave function $\Psi$ expansion, the size of the pseudostate basis set was chosen as $N^{\prime}=\frac{3}{2} N$, which appeared to be sufficient for most of the $t$ points. There were also additional factors taken into account for the accurate representation of the resolvent in Eq. (22). The powers of the internuclear coordinate $r_{01}$, analogously to the wave function, were restricted to even integers and were generated randomly for each basis function from the log-normal distribution within the $0-80$ range. However, for small values of $t(\leq 0.1)$, due to a cancellation in the numerator of Eq. (21), an additional tuning of the distribution was made and $N^{\prime}=2 N$ was set to achieve high accuracy. Moreover, in this critical region of small $t$, the function $f(t)$ was expanded in a power series in Eq. (23), and the higher order expansion terms were obtained from the fit to numerical values of $f(t)$. In order to perform the integration in Eq. (21), we used a polynomial interpolation of the integrand for $t>0.1$, and a power expansion for the critical region $t \in[0,0.1]$.

The convergence of the Bethe logarithm with the increasing size of the naECG basis is shown in Table III Six significant figures can be considered stable and the estimated relative uncertainty is a half ppm for all molecules, as previously for $\mathrm{H}_{2}$.

\section{HIGHER ORDER QED}

The higher order QED corrections are calculated within the Born-Oppenheimer approximation. First let us consider the second iteration of the relativistic correction $\mathcal{E}^{(4)}$ to the $\mathrm{BO}$ potential

$$
E_{\mathrm{sec}}^{(6)}=\left\langle\chi(R)\left|\mathcal{E}^{(4)}(R) \frac{1}{\left(E^{(2)}-H_{\mathrm{n}}\right)^{\prime}} \mathcal{E}^{(4)}(R)\right| \chi(R)\right\rangle,
$$

where $\chi(R)$ is the radial nuclear wave function obtained from the radial Schrödinger equation with the Hamiltonian consisting of the nuclear kinetic energy and the nonrelativistic BO potential. This term is of $\alpha^{6}$ order and is considered separately for consistency with the previous calculations of $E^{(4)}$ using the nonadiabatic perturbation theory. The main $\alpha^{6}$ contribution is obtained by averaging the $\mathcal{E}^{(6)}(R)$ potential obtained in the BO framework in Ref. [21] and the unknown $1 / \mu$ correction is estimated to be smaller than the numerical uncertainty of $\mathcal{E}^{(6)}(R)$.

Because of the significant increase in the accuracy of the QED correction achieved in this work, the dominating contribution to the uncertainty comes from the higher order $E^{(7)}$ correction. Currently, an explicit form of this correction is unknown, which prevents its accurate evaluation. Its first estimation, made within the $\mathrm{BO}$ approximation framework, was reported in Ref. [21]. Here, following [7], we account for several additional terms, namely

$$
\begin{aligned}
E^{(7)} \approx & \pi\left\langle\sum_{a, x} \delta^{3}\left(r_{a x}\right)\right\rangle\left\{\frac { 1 } { \pi } \left[A_{60}+A_{61} \ln \alpha^{-2}\right.\right. \\
& \left.\left.+A_{62} \ln ^{2} \alpha^{-2}\right]+\frac{B_{50}}{\pi^{2}}+\frac{C_{40}}{\pi^{3}}\right\}-E_{0}^{(7)}-E_{1}^{(7)}
\end{aligned}
$$


TABLE III. Convergence of the Bethe logarithm $\ln k_{0}$ with the increasing size $N$ of the naECG basis set. The final uncertainty for $\ln k_{0}$ is due to numerical inaccuracy of $f(t)$ at small $t$.

\begin{tabular}{cccccc}
\hline \hline$N$ & $\mathrm{D}_{2}$ & $\mathrm{~T}_{2}$ & HD & HT & DT \\
\hline 128 & 3.01614565 & 3.01655724 & 3.01800962 & 3.01817520 & 3.01748026 \\
256 & 3.01828811 & 3.01832328 & 3.01820759 & 3.01833511 & 3.01825913 \\
512 & 3.01845913 & 3.01848717 & 3.01834702 & 3.01837325 & 3.01843911 \\
1024 & 3.01847332 & 3.01851461 & 3.01838584 & 3.01841464 & 3.01848483 \\
2048 & 3.01847598 & 3.01851989 & 3.01839311 & 3.01841833 & 3.01849612 \\
$\infty$ & $3.018478(2)$ & $3.018522(3)$ & $3.018397(4)$ & $3.018422(4)$ & $3.018501(5)$ \\
\hline \hline
\end{tabular}

where $A, B$, and $C$ coefficients corresponds to the well known one- , two-, and three-loop hydrogenic Lamb shift [10]. Since we calculate the dissociation energy, the atomic values $E_{0,1}^{(7)}$ are subtracted out.

Finally, at the achieved accuracy level, the nuclear finite size effect cannot be neglected and it is accounted for by the following formula

$$
E_{\mathrm{FS}}^{(4)}=\alpha^{4} \frac{2 \pi}{3}\left\langle\sum_{a, x} \delta^{3}\left(r_{a x}\right)\right\rangle \frac{\left(r_{c 0}^{2}+r_{c 1}^{2}\right)}{2 \lambda^{2}}-E_{\mathrm{FS} 0}^{(4)}-E_{\mathrm{FS} 1}^{(4)},
$$

where $r_{c 0 / 1}^{2}$ is the mean square charge radius of the nucleus $0 / 1, \lambda$ is the electron Compton wavelength, and atomic values $E_{\mathrm{FS} 0,1}^{(4)}$ are subtracted out.

\section{FINAL RESULTS AND CONCLUSION}

Theoretical predictions for the known contributions to the dissociation energies of all molecular hydrogen isotopologues are presented in Table IV] Thanks to the direct nonadiabatic calculation of the nonrelativistic energy $[23-25]$ and also of the relativistic [13, 26, 27] and leading quantum electrodynamic corrections, the theoretical dissociation energy of all the isotopologues of molecular hydrogen has reached the level of $0.8 \mathrm{MHz}\left(26 \times 10^{-6} \mathrm{~cm}^{-1}\right.$ or $8 \times 10^{-10}$ of relative uncertainty). The higher order $m \alpha^{6}$ QED contribution has been calculated [21] within the BO approximation, but the corresponding uncertainty is almost negligible. At present the accuracy of theoretical predictions is limited by the poorly known $E^{(7)}$ term of the $\alpha$-expansion (1), which has been estimated using the atomic hydrogen values with $25 \%$ uncertainty, as in Ref. [7]. As a result, the significantly improved theoretical predictions for the ground state dissociation energy of the $\mathrm{D}_{2}$ molecule (as well as for $\mathrm{H}_{2}$ ) are in very good agreement with the most recent measurement [22], but the experimental uncertainty is more than 20 times larger than the theoretical one. The situation is more intriguing for the dissociation energy of the HD molecule. Our theoretical prediction differs by $3 \sigma$ from the most recent measurement in Ref. [4]. If this experimental value is confirmed, this could indicate the existance of yet unknown physical effects, which are specific to heteronuclear molecules only.
Acknowledgments - We thank referees for their pertinent suggestions which helped to improve the paper. This research was supported by National Science Center (Poland) Grants No. 2014/15/B/ST4/05022 (M.P.) and 2017/25/B/ST4/01024 (J.K.) as well as by a computing grant from Poznań Supercomputing and Networking Center and by PL-Grid Infrastructure.

[1] C. Cheng, J. Hussels, M. Niu, H. L. Bethlem, K. S. E. Eikema, E. J. Salumbides, W. Ubachs, M. Beyer, N. J. Hölsch, J. A. Agner, et al., Phys. Rev. Lett. 121, 013001 (2018).

[2] N. Hölsch, M. Beyer, E. J. Salumbides, K. S. E. Eikema, W. Ubachs, C. Jungen, and F. Merkt, Phys. Rev. Lett. 122, 103002 (2019).

[3] J. Liu, D. Sprecher, C. Jungen, W. Ubachs, and F. Merkt, J. Chem. Phys. 132, 154301 (2010).

[4] D. Sprecher, J. Liu, C. Jungen, W. Ubachs, and F. Merkt, J. Chem. Phys. 133, 111102 (2010).

[5] T. M. Trivikram, M. Schlösser, W. Ubachs, and E. J. Salumbides, Phys. Rev. Lett. 120, 163002 (2018).

[6] K.-F. Lai, P. Czachorowski, M. Schlosser, M. Puchalski, J. Komasa, K. Pachucki, W. Ubachs, and E. J. Salumbides, Phys. Rev. Lett. (2019), submitted.

[7] M. Puchalski, J. Komasa, P. Czachorowski, and K. Pachucki, Phys. Rev. Lett. 122, 103003 (2019).

[8] R. K. Altmann, L. S. Dreissen, E. J. Salumbides, W. Ubachs, and K. S. E. Eikema, Phys. Rev. Lett. 120, 043204 (2018).

[9] J. Hussels, C. Cheng, H. L. Bethlem, K. S. E. Eikema, E. Salumbides, W. Ubachs, M. Beyer, N. Hlsch, F. Merkt, $\mathrm{S}$. Hu, et al., in International Conference on Precision Physics and Fundamental Physical Constants, Tihany, Hungary (2019).

[10] M. I. Eides, H. Grotch, and V. A. Shelyuto, Phys. Rep. 342, 63 (2001).

[11] K. Pachucki and J. Komasa, J. Chem. Phys. 144, 164306 (2016).

[12] D. B. Kinghorn and L. Adamowicz, Phys. Rev. Lett. 83, 2541 (1999).

[13] M. Puchalski, A. Spyszkiewicz, J. Komasa, and K. Pachucki, Phys. Rev. Lett. 121, 073001 (2018).

[14] T. Kato, T., Commun. Pure Appl. Math. 10, 151, (1957).

[15] K. Pachucki, Phys. Rev. Lett. 106, 193007 (2011).

[16] G. W. F. Drake and R. A. Swainson, Phys. Rev. A 41, 1243 (1990).

[17] R. Pohl, A. Antognini, F. Nez, F. D. Amaro, F. Biraben, J. M. R. Cardoso, D. S. Covita, A. Dax, S. Dhawan, L. M. P. Fernandes, et al., Nature 466, 213 (2010). 
TABLE IV. Theoretical predictions for the dissociation energy budget for the ground level of the molecular hydrogen isotopologues. $E_{\mathrm{FS}}$ is the finite nuclear size correction with $r_{p}=0.8414(19) \mathrm{fm}$ [28], $r_{d}=2.12799(74) \mathrm{fm}$ [28], and $r_{t}=1.7591(363)$ fm [29]. All the energy entries are given in $\mathrm{cm}^{-1}$.

\begin{tabular}{lccccc}
\hline \hline Contribution & $\mathrm{D}_{2}$ & $\mathrm{~T}_{2}$ & HD & HT & DT \\
\hline$E^{(2)}$ & $36749.09099000(1)$ & $37029.22486700(1)$ & $36406.51089007(1)$ & $36512.92800911(1)$ & $36882.00984348(1)$ \\
$E^{(4)}$ & $-0.52820605(9)$ & $-0.5267500(2)$ & $-0.5298875(2)$ & $-0.5293779(2)$ & $-0.5275236(3)$ \\
$E^{(5)}$ & $-0.198256(3)$ & $-0.199735(4)$ & $-0.196441(4)$ & $-0.197005(5)$ & $-0.198958(5)$ \\
$E^{(6)}$ & $-0.002096(6)$ & $-0.002110(6)$ & $-0.002080(6)$ & $-0.002085(6)$ & $-0.002103(6)$ \\
$E_{\text {sec }}^{(6)}$ & 0.0000094 & 0.0000094 & 0.0000093 & 0.0000093 & 0.0000094 \\
$E^{(7)}$ & $0.000103(25)$ & $0.000103(25)$ & $0.000102(25)$ & $0.000102(25)$ & $0.000103(25)$ \\
$E_{\mathrm{FS}}^{(4)}$ & -0.000202 & $-0.000139(6)$ & -0.000116 & $-0.000084(3)$ & $-0.000171(3)$ \\
Total & $36748.362342(26)$ & $37028.496245(27)$ & $36405.782477(26)$ & $36512.199569(26)$ & $36881.281200(26)$ \\
Exp. & $36748.36286(68)$ & & $36405.78366(36)$ & & \\
Diff. & $0.00052(68)$ & & $0.00118(36)$ & & \\
\hline \hline
\end{tabular}

[18] K. Pachucki and J. Komasa, Phys. Rev. Lett. 92, 213001 (2004).

[19] B.-L. Zhou, J.-M. Zhu, and Z.-C. Yan, Phys. Rev. A 73, 014501 (2006).

[20] V. I. Korobov, Phys. Rev. A 85, 042514 (2012).

[21] M. Puchalski, J. Komasa, P. Czachorowski, and K. Pachucki, Phys. Rev. Lett. 117, 263002 (2016).

[22] J. Liu, E. J. Salumbides, U. Hollenstein, J. C. J. Koelemeij, K. S. E. Eikema, W. Ubachs, and F. Merkt, J. Chem. Phys. 130, 174306 (2009).

[23] K. Pachucki and J. Komasa, Phys. Chem. Chem. Phys. 20, 247 (2018).

[24] K. Pachucki and J. Komasa, Phys. Chem. Chem. Phys. 20, 26297 (2018).

[25] K. Pachucki and J. Komasa, Phys. Chem. Chem. Phys. 21, 10272 (2019).
[26] L. M. Wang and Z.-C. Yan, Phys. Rev. A 97, 060501 (2018).

[27] L. Wang and Z.-C. Yan, Phys. Chem. Chem. Phys. 20, 23948 (2018).

[28] CODATA 2018 recommended values, https://physics.nist.gov/cuu/Constants

[29] I. Angeli, K.P. Marinova, At. Data Nucl. Data Tables 99, 69 (2013)

\section{Expectation values of individual operators}

In Table $\mathrm{V}$ we present nonrelativistic energies and expectation values (in a.u.) of individual operators with the nonrelativistic 4-body naECG wave function for a possible comparison with any future calculations. 
TABLE V. Mean values of various operators with naECG wave function for the ground molecular state

\begin{tabular}{|c|c|c|c|c|c|}
\hline Operator & $\mathrm{D}_{2}$ & $\mathrm{~T}_{2}$ & HD & HT & DT \\
\hline $\mathrm{H}$ & $-1.16716880926(4)$ & $-1.16853567559(17)$ & $-1.16547192393(6)$ & $-1.16600203724(12)$ & $-1.16781967331(16)$ \\
\hline$\vec{J}^{2}$ & $2.52756521816(15)$ & 5) $2.5317141635(6)$ & $2.5225064462(3)$ & $2.5240761103(5)$ & $2.5295324383(8)$ \\
\hline$\sum_{a} 4 \pi \delta\left(r_{0 a}\right)$ & $5.70364695(5)$ & $5.7166985(2)$ & $5.68510231(6)$ & $5.68949373(8)$ & $5.7091406(3)$ \\
\hline$\sum_{a} 4 \pi \delta\left(r_{1 a}\right)$ & $5.70364695(5)$ & $5.7166985(2)$ & $5.68949506(7)$ & $5.69535191(11)$ & $5.7106075(4)$ \\
\hline $4 \pi \delta\left(r_{23}\right)$ & $0.205013236(5)$ & $0.20596494(3)$ & $0.203833913(15)$ & $0.20420205(2)$ & $0.20546614(3)$ \\
\hline$\sum_{a} p_{a}^{4}$ & $-13.0767572(3)$ & $-13.1061977(9)$ & $13.0395620(3)$ & $-13.0512614(5)$ & $-13.0908344(7)$ \\
\hline$p_{2}^{i}\left(\frac{\delta^{i j}}{r_{23}}+\frac{r_{23}^{i} r_{23}^{j}}{r_{23}^{3}}\right) p_{3}^{j}$ & $0.093386335(4)$ & $0.09372850(2)$ & $0.09295918(4)$ & $0.093093113(14)$ & $0.093549582(9)$ \\
\hline$\sum_{a} p_{0}^{i}\left(\frac{\delta^{i j}}{r_{0 a}}+\frac{r_{0 a}^{i} r_{0 a}^{j}}{r_{0 a}^{3}}\right)$ & $p_{a}^{j}-2.50716722(8)$ & $-2.5126573(3)$ & $-2.4957024(3)$ & $-2.4960339(4)$ & $-2.5078556(5)$ \\
\hline$\sum_{a} p_{1}^{i}\left(\frac{\delta^{i j}}{r_{1 a}}+\frac{r_{1 a}^{i} r_{1 a}^{j}}{r_{1 a}^{3}}\right)$ & $p_{a}^{j}-2.50716722(8)$ & $-2.5126573(3)$ & $-2.5048648(3)$ & $-2.5088521(4)$ & $-2.5117170(5)$ \\
\hline$p_{0}^{i}\left(\frac{\delta^{i j}}{r_{01}}+\frac{r_{01}^{i} r_{01}^{j}}{r_{01}^{3}}\right) p_{1}^{j}$ & $-17.8021387(4)$ & $-21.9643326(11)$ & $-14.3880335(7)$ & $-15.3048141(15)$ & $-19.5509683(15)$ \\
\hline$\sum_{a}\left\langle r_{0 a}^{-3}\right\rangle_{\epsilon}$ & $-3.6146879(8)$ & $-3.622724(3)$ & $-3.6022426(8)$ & $-3.6047229(11)$ & $-3.617784(2)$ \\
\hline$\sum_{a}\left\langle r_{1 a}^{-3}\right\rangle_{\epsilon}$ & $-3.6146879(8)$ & $-3.622724(3)$ & $-3.6066881(7)$ & $-3.6106491(8)$ & $-3.619274(3)$ \\
\hline$\left\langle r_{23}^{-3}\right\rangle_{\epsilon}$ & $0.40552277(4)$ & $0.40709024(16)$ & $0.4035864(5)$ & $0.4041904(11)$ & $0.4062689(9)$ \\
\hline$\sum_{a}\left\langle r_{0 a}^{-4}\right\rangle_{\epsilon}$ & $-2.86527(2)$ & $-2.86932(5)$ & $-2.87197(3)$ & $-2.87783(4)$ & $-2.89529(4)$ \\
\hline$\sum_{a}\left\langle r_{1 a}^{-4}\right\rangle_{\epsilon}$ & $-2.86527(2)$ & $-2.86932(5)$ & $-2.87551(3)$ & $-2.88262(4)$ & $-2.89650(4)$ \\
\hline$\sum_{a<b} \frac{\vec{r}_{0 a}}{r_{0 a}^{3}} \cdot \frac{\vec{r}_{0 b}}{r_{0 b}^{3}}$ & $-0.0116611(7)$ & $-0.011686(3)$ & $-0.0115805(16)$ & $-0.0115713(5)$ & $-0.0116539(4)$ \\
\hline$\sum_{a<b} \frac{\vec{r}_{0 a}}{r_{0 a}^{3}} \cdot \frac{\vec{r}_{1 b}}{r_{1 b}^{3}}$ & $-0.234951712(6)$ & $-0.23654076(3)$ & $-0.232986687(5)$ & $-0.233599584(7)$ & $-0.23570773(3)$ \\
\hline$\sum_{a<b} \frac{\vec{r}_{1 a}}{r_{1 a}^{3}} \cdot \frac{\vec{r}_{1 b}}{r_{1 b}^{3}}$ & $-0.0116611(6)$ & $-0.011686(3)$ & $-0.0116778(2)$ & $-0.0117047(5)$ & $-0.0116877(4)$ \\
\hline$\sum_{a} \frac{\vec{r}_{01}}{r_{01}^{3}} \cdot \frac{\vec{r}_{0 a}}{r_{0 a}^{3}}$ & $-1.17353(6)$ & $-1.1774(2)$ & $-1.16784(9)$ & $-1.16892(12)$ & $-1.17456(10)$ \\
\hline$\sum_{a} \frac{\vec{r}_{01}}{r_{01}^{3}} \cdot \frac{\vec{r}_{1 a}}{r_{1 a}^{3}}$ & $1.17353(6)$ & $1.1774(2)$ & $1.16950(8)$ & $1.17116(11)$ & $1.17513(11)$ \\
\hline
\end{tabular}

